\title{
Growth and Yield of Irrigated Bt Cotton (Gossypium hirsutum L.) as Influenced by Different Agronomic Practices
}

\author{
Basavaraj*, B.M. Chittapur, A.S. Halepyati, A. Ameregouda, \\ G.S. Yadahalli and M.Y. Ajayakumar \\ Department of Agronomy, University of Agricultural Sciences, Raichur-584102, Karnataka, India \\ *Corresponding author
}

\section{A B S T R A C T}

\begin{tabular}{|c|}
\hline Keywords \\
\hline $\begin{array}{l}\text { Bt cotton, Foliar } \\
\text { spray, } \mathrm{KH}_{2} \mathrm{PO}_{4} \text {, } \\
\text { Major and } \\
\text { micronutrients, } \\
\text { Polythene } \\
\text { mulching. }\end{array}$ \\
\hline Article Info \\
\hline $\begin{array}{l}\text { Accepted: } \\
\text { 04 October } 2017 \\
\text { Available Online: } \\
10 \text { December } 2017\end{array}$ \\
\hline
\end{tabular}

A field experiment was conducted to study the influence of different agronomic practices on growth, yield attributes and yield of $B t$ cotton at the Main Agricultural Research Station, Raichur during Kharif 2016. Nine treatments comprising of normal spaced and fertilized non- $B t$ (cv. RAHH-455) and $B t$ hybrid (cv. Bindas) in addition to closer spacing $B t$ hybrid supplied with $125 \%$ of recommended nutrients (187.5: 92.75: $92.75 \mathrm{~kg}$ $\mathrm{N}: \mathrm{P}_{2} \mathrm{O}_{5}: \mathrm{K}_{2} \mathrm{O}$ ha $\left.^{-1}\right)$, foliar spray of $1 \% \mathrm{MgSO}_{4}$ twice and $1 \% \mathrm{KNO}_{3}$ thrice, soil application of $\mathrm{MgSO}_{4}\left(25 \mathrm{~kg} \mathrm{ha}^{-1}\right)$ and $\mathrm{FeSO}_{4}\left(10 \mathrm{~kg} \mathrm{ha}^{-1}\right)$ and $\mathrm{ZnSO}_{4}\left(10 \mathrm{~kg} \mathrm{ha}^{-1}\right)$, foliar spray of $1 \%$ $\mathrm{MgSO}_{4}+1 \%$ 19:19:19 or $1 \% \mathrm{KH}_{2} \mathrm{PO}_{4}$ and polyethylene mulching replicated thrice. The results revealed that closer spaced $B t$ hybrid $+125 \% \mathrm{RDF}+3$ sprays of $1 \%$ each of $\mathrm{MgSO}_{4}$ and 19:9:19 and polyethylene mulching recorded significantly higher plant height $(159.2 \mathrm{~cm})$, monopodia (2.0), sympodia (27.7), total dry matter production $(347.0 \mathrm{~g})$, leaf area index (1.81), number of bolls plant ${ }^{-1}(48.27)$ and seed cotton yield (4122 kg ha $\left.{ }^{-1}\right)$ compared to other nutritional treatments. It also registered higher net returns ( $\square 1,59,296$ $\left.\mathrm{ha}^{-1}\right)$, gross returns $(\square 2,22,588)$ and $\mathrm{B}: \mathrm{C}$ ratio (3.52). It was followed by closer spaced $B t$ hybrid + foliar spray of $1 \% \mathrm{KH}_{2} \mathrm{PO}_{4}$ thrice.

\section{Introduction}

Cotton (Gossypium hirsutum L.), is considered as an important fibre crop of India and Karnataka. It is the backbone of textile industries providing $85 \%$ of raw material to textile industry and it earns about 33 per cent of total foreign exchange (Anon., 2014). Most of the $B t$ cotton hybrids bear fruiting parts by 20 to 25 days and also mature early in comparison to the erstwhile inter specific hybrids and hence need for optimum nutrition begins from the beginning in cotton otherwise there would be flower and/or boll drop, early leaf reddening etc. which greatly affect overall crop production. Being early and semi-determinate nature the rate of growth and rejuvenation capacity of the plant after first flush of flowering is slow and therefore there is more nutritional requirement after first bearing and this necessitates additional nutrition (Mamatha et al., 2009). Further, the deficiency of micronutrients has become major constraint in productivity, stability and sustainability of cotton ecosystem now than ever before (Yadav and Meena, 2009).

Recently, plastic mulch is becoming popular in vegetable production under water constraints. Plastic (PE) mulches improve 
physical, chemical and biological properties of soil and boost soil temperature and thereby enhance root activity, which is critical during winter in crops like cotton. Mulching reported to enhance yield by 50 - $60 \%$ over no mulching under rainfed situations (Dilip Kumar et al., 1990 and Rajput and Singh, 1970), which is an important consideration even in irrigation commands due to uncertainty rain and poor inflow to the reservoirs.

In Karnataka, North-eastern and Northern dry zone drenched with UKP and TBP irrigation commands account for major share in area and production of cotton. Though yields as high as 15 to $18 \mathrm{q} \mathrm{ac}^{-1}$ were realized after the advent of $B t$ cultivars, of late crop yields are either stagnating or declining due to many reasons raising concerns of all stake holders. Therefore, keeping above points in view a field experiment on yield maximization in cotton comprising of cultivars, plant density, enhanced primary and foliar nutrition, and polyethylene (PE) mulching in TBP irrigation command was envisaged during 2016-17 under irrigated condition.

\section{Materials and Methods}

The field experiment was conducted during kharif 2016 and at the Main Agricultural Research Station Raichur, Karnataka. The experiment laid out in Randomized Complete Block Design (RCBD) with three replications comprised of 9 treatments viz., $\mathrm{T}_{1^{-}}$Normal spaced and fertilized $(90 \mathrm{~cm} \mathrm{X} 60 \mathrm{~cm})$ non- $B t$ hybrid cv.RAHH-455, T2- Normal spaced and fertilized $B t$ hybrid (cv. Bindas), $\mathrm{T}_{3}$ - Closer spacing $(90 \mathrm{~cm} \mathrm{X} 45 \mathrm{~cm}) B t$ hybrid (cv. Bindas), $\mathrm{T}_{4^{-}} \mathrm{T}_{3}+125 \%$ of recommended nutrients (187.5: 92.75: $92.75 \mathrm{~kg} \mathrm{NPK} \mathrm{ha}^{-1}$ ), $\mathrm{T}_{5}-\mathrm{T}_{4}+$ Recommended foliar spray (1\% spray of $\mathrm{MgSO}_{4}$ at 90 and 110 days after sowing (DAS) and $1 \%$ spray of $\mathrm{KNO}_{3}$ during flowering, boll initiation stage), $\mathrm{T}_{6}-\mathrm{T}_{4}+$ Soil application of micronutrients $\mathrm{MgSO}_{4} @ 25 \mathrm{~kg}$ $\mathrm{ha}^{-1}, \mathrm{FeSO}_{4} @ 10 \mathrm{~kg}$ and $10 \mathrm{~kg} \mathrm{ZnSO} 4 \mathrm{ha}^{-1}$, $\mathrm{T}_{7^{-}} \mathrm{T}_{4}+$ three sprays of $1 \% \mathrm{MgSO}_{4}+1 \%$ 19:19:19 during 60-65, 80-85 and 100-105 DAS, $\mathrm{T}_{8^{-}} \mathrm{T}_{4}+$ foliar application of @ $1 \%$ $\mathrm{KH}_{2} \mathrm{PO}_{4}$ at 60,80 and100 DAS, $\mathrm{T}_{9}-\mathrm{T}_{7}+$ Soil mulching through polythene sheet (200 gauge). Sowing was done by hand dibbling and recommended fertilizer was applied using urea, DAP and muriate of potash. Data on growth and yield parameters were recorded from five randomly selected plants in each treatment; seed cotton yield $\left(\mathrm{kg}\right.$ plot $\left.^{-1}\right)$ was calculated from whole plot and converted into $\mathrm{kg} \mathrm{ha}^{-1}$, besides economics was worked out.

\section{Results and Discussion}

\section{Growth Parameters and Seed cotton yield}

The plant height, number of monopodial branches plant $^{-1}$, number of sympodial branches plant $^{-1}$ and total dry matter production plant $^{-1}$ differed significantly due to different yield maximization practices (Table 1). The data revealed that closer spaced $B t$ hybrid $+125 \%$ RDF + three sprays of $1 \%$ each of $\mathrm{MgSO}_{4}$ and 19:19:19 and polyethylene (PE) mulching $\left(\mathrm{T}_{9}\right)$ recorded significantly higher plant height $(159.2 \mathrm{~cm})$, monopodial plant ${ }^{-1}(2.0)$, sympodia plant $^{-1}$ (27.7), leaf area index (1.81) and total dry matter production (347.0 $\mathrm{g}$ plant $^{-1}$ ) compared to other treatments. It was followed by closer spaced $B t$ hybrid $+125 \% \mathrm{RDF}+$ foliar application of $\mathrm{KH}_{2} \mathrm{PO}_{4} @ 1 \%$ at 60, 80 and 100 DAS. Thus, these significant increases in all the growth parameters with increased fertilization in combination with foliar sprays of major and micro nutrients and PE mulching were responsible for higher assimilates production and their translocation to sink. Similar findings were reported by Sawan et al., (2006), Sakarvadia et al., (2009), Saleem et al., (2010), Kaur et al., (2010), Biradar (2011) and Hosamani et al., (2013) who also 
reported improved growth parameters and consequently cotton yield with higher nutrition and foliar spray of liquid soluble fertilizers over recommended practice.

Significant differences occurred in leaf reddening index (LRI) at different stages of development except at 90 DAS (Table 3). Non $-B t$ cv. RAHH 455 with normal spacing/ population recorded the maximum LRI at all the stages $(0.71,2.33$ and 2.93 at 90 and 135 DAS and at final picking, respectively) among all closely followed by similar spaced $B t$ cultivar: others were also at par except $\mathrm{T}_{9}$ (0.20, 1.07 and 1.93 at 90 and 135 DAS and at final picking) with around half the values of the highest recording treatment had lower LR indices among all.

Further, among the leaf reddening management (LRM) interventions, soil supplementation of micronutrients comprising of $\mathrm{MgSO}_{4} @ 25 \mathrm{~kg} \mathrm{ha}^{-1}$ and $\mathrm{FeSO}_{4}$ and $\mathrm{ZnSO}_{4}$ each at $10 \mathrm{~kg} \mathrm{ha}^{-1}$ increased yield by $405 \mathrm{~kg} \mathrm{ha}^{-1}$ over application of $125 \% \mathrm{RDF}$ with $12 \%$ increase in yield. This was also found superior to foliar application of $\mathrm{MgSO}_{4}$ and $\mathrm{KNO}_{3}$ twice each.

Recent recommendation of $1 \%$ each of $\mathrm{MgSO}_{4}$ and 19:19:19 all thrice at flower initiation, boll development and boll bursting in the state resulted in and yield improvement by 509 and $1272 \mathrm{~kg} \mathrm{ha}^{-1}$ (16 and $51 \%$ more yield), respectively over application of $125 \%$ $\mathrm{RDF}$ and RDF and recommended spacing alone. The results are in conformity with Shivamurthy and Biradar (2014).

In the investigation, significantly higher number of total bolls per plant (48.27), boll weight $(5.64 \mathrm{~g})$ and TDM production $(347.0 \mathrm{~g}$ plant $^{-1}$ ) were recorded with closer spacing with $125 \%$ RDF and three sprays of $1 \%$ each of $\mathrm{MgSO}_{4}$ and 19:19:19 at 60, 80 and 100 DAS with PE mulching over normal spaced non- $B t$ cv. RAHH-445 (35.37, 4.94g, 270g respectively total number of bolls, boll weight plant $^{-1}$ and TDM respectively). As a consequence of improved growth and yield components, Closer spaced $B t$ hybrid $+125 \%$ $\mathrm{RDF}+$ three sprays (60-65,80-85,100-105 days after sowing) of $1 \%$ each of $\mathrm{MgSO}_{4}$ and 19:19:19 and polyethylene (PE) mulching $\left(\mathrm{T}_{9}\right)$ registered significantly higher seed cotton yield $\left(4,122 \mathrm{~kg} \mathrm{ha}^{-1}\right)$ compared to other treatments (Table 2). It was closely followed by closer spaced $B t$ hybrid $+125 \% \mathrm{RDF}+$ foliar application of $\mathrm{KH}_{2} \mathrm{PO}_{4} @ 1 \%$ at 60, 80 and 100 days after sowing (3912 kg ha-1), closer spacing + three sprays of $1 \% \mathrm{MgSO}_{4}$ and 19:19:19 (3,777 $\left.\mathrm{kg} \mathrm{ha}^{-1}\right)$ and closer spaced $B t+125 \% \mathrm{RDF}+$ Soil application of micronutrients @ $25 \mathrm{~kg} \mathrm{MgSO}_{4}+10 \mathrm{~kg}$ $\mathrm{FeSO}_{4}+10 \mathrm{~kg} \mathrm{ZnSO}_{4} \mathrm{ha}^{-1}\left(3,673 \mathrm{~kg} \mathrm{ha}^{-1}\right)$.

Kaur et al., (2010), Biradar et al., (2012) and Basavanneppa et al., (2011) also observed higher yield and yield attributing characters and TDM production with higher doses of fertilizer compared to lower doses and recommended fertilizer practice. Soil and foliar application of $\mathrm{MgSO}_{4}$ also increased seed cotton yield because of magnesium which is an integral part of chlorophyll, which increased chlorophyll content and its stability and thereby photosynthesis and seed cotton yield. The results are in conformity with the findings of Brar et al., (2008), Rajendran et al., (2011) and Hosmath (2011). $\mathrm{KH}_{2} \mathrm{PO}_{4}$ might have alleviated the inhibitive effects of climatic impacts of winter on cotton besides improving supply of $\mathrm{P}$ and $\mathrm{K}$.

The superiority of PE mulch mainly lies in its effect in maintaining soil moisture balance and raising soil temperature as observed during October as shown in Figure 1, which probably prevailed throughout winter and specially during reproductive cycle of plant thereby invigorated root activity that complemented plant rhizosphere need. 
Table.1 Growth attributes of $B t$ cotton as influenced by different agronomic practices

\begin{tabular}{|c|c|c|c|c|c|c|c|c|}
\hline \multirow[t]{2}{*}{ Treatment } & \multirow{2}{*}{$\begin{array}{l}\text { Plant height } \\
(\mathrm{cm})\end{array}$} & \multirow{2}{*}{$\begin{array}{l}\text { Monopodia } \\
\text { plant }^{-1}\end{array}$} & \multirow{2}{*}{$\begin{array}{l}\text { Sympodia } \\
\text { plant }^{-1}\end{array}$} & \multicolumn{4}{|c|}{ Leaf area index } & \multirow{2}{*}{$\begin{array}{l}\text { TDM } \\
\text { (g plant) }\end{array}$} \\
\hline & & & & 45 DAS & 90 DAS & 135 DAS & $\begin{array}{l}\text { At final } \\
\text { picking }\end{array}$ & \\
\hline $\begin{array}{l}\mathrm{T}_{1}: \text { Normal spaced }(90 \mathrm{~cm} \text { X } 60 \mathrm{~cm}) \text { non- } \\
B t \text { hybrid cv.RAHH- } 455 .\end{array}$ & $142.0^{\mathrm{b}}$ & $2.2^{\mathrm{a}}$ & $18.0^{\mathrm{e}}$ & $8.51^{\mathrm{d}}$ & $66.83^{\mathrm{b}}$ & $96.89^{b}$ & $1.18^{\mathrm{b}}$ & $270.0^{\mathrm{e}}$ \\
\hline $\begin{array}{l}\mathrm{T}_{2}: \text { Normal planting }(90 \mathrm{~cm} \mathrm{X} 60 \mathrm{~cm}) \mathrm{Bt} \\
\text { hybrid Bindas }\end{array}$ & $144.5^{\mathrm{ab}}$ & $2.2^{\mathrm{a}}$ & $23.8^{\mathrm{cd}}$ & $10.35^{\mathrm{bc}}$ & $70.18^{\mathrm{ab}}$ & $98.64^{\mathrm{ab}}$ & $1.64^{\mathrm{a}}$ & $299.1^{\mathrm{cd}}$ \\
\hline $\begin{array}{l}\mathrm{T}_{3} \text { : Closer spacing }(90 \mathrm{~cm} \mathrm{X} 45 \mathrm{~cm}) \mathrm{Bt} \\
\text { hybrid Bindas }\end{array}$ & $145.9^{\mathrm{ab}}$ & $1.8^{\mathrm{a}}$ & $21.5^{\mathrm{d}}$ & $9.48^{\mathrm{cd}}$ & $69.23^{\mathrm{ab}}$ & $98.36^{\mathrm{ab}}$ & $1.22^{\mathrm{b}}$ & $293.2^{d}$ \\
\hline $\begin{array}{l}\mathrm{T}_{4}: \mathrm{T}_{3}+125 \% \text { of Rec. Nutrients (187.5: } \\
\left.\text { 92.75: } 92.75 \mathrm{~kg} \mathrm{~N}: \mathrm{P}_{2} \mathrm{O}_{5}: \mathrm{K}_{2} \mathrm{O} / \mathrm{ha}\right)\end{array}$ & $146.7^{\mathrm{ab}}$ & $1.6^{\mathrm{a}}$ & $23.9^{\mathrm{cd}}$ & $10.66^{\mathrm{b}}$ & $70.64^{\mathrm{ab}}$ & $99.12^{\mathrm{ab}}$ & $1.66^{\mathrm{a}}$ & $305.9^{\mathrm{cd}}$ \\
\hline $\begin{array}{l}\mathrm{T}_{5}: \mathrm{T}_{4}+\text { Recommended foliar spray ( } 1 \% \\
\text { spray } \mathrm{MgSO}_{4} \text { at } 90 \& 110 \mathrm{DAS} \text { and } \\
1 \% \text { spray of } \mathrm{KNO}_{3} \text { during flowering \& } \\
\text { Boll initiation stage) }\end{array}$ & $155.1^{\mathrm{ab}}$ & $2.2^{\mathrm{a}}$ & $24.1^{\mathrm{cd}}$ & $11.19^{\mathrm{ab}}$ & $70.83^{\mathrm{ab}}$ & $100.21^{\mathrm{ab}}$ & $1.69^{\mathrm{a}}$ & $312.5^{b-c}$ \\
\hline $\begin{array}{l}\mathrm{T}_{6}: \mathrm{T}_{4}+\text { Soil application of Micronutrients } \\
\text { @ } 25 \mathrm{~kg} \mathrm{MgSO}_{4} / \mathrm{ha}+10 \mathrm{~kg} \mathrm{FeSO}+10 \\
\mathrm{~kg} \mathrm{ZnSO}_{4} / \mathrm{ha} \text {. }\end{array}$ & $156.7^{\mathrm{a}}$ & $1.9^{\mathrm{a}}$ & $24.6^{\mathrm{bc}}$ & $11.35^{\mathrm{ab}}$ & $71.59^{\mathrm{ab}}$ & $101.17^{\mathrm{ab}}$ & $1.72^{\mathrm{a}}$ & $319.0^{\mathrm{bc}}$ \\
\hline $\begin{array}{l}\mathrm{T}_{7}: \mathrm{T}_{4}+\text { three sprays of } 1 \% \mathrm{MgSO}_{4}+1 \% \\
19: 19: 19 \text { during } 60-65,80-85 \& 100- \\
105 \text { DAS }\end{array}$ & $157.7^{\mathrm{a}}$ & $2.1^{\mathrm{a}}$ & $25.6^{\mathrm{a}-\mathrm{c}}$ & $11.93^{\mathrm{a}}$ & $72.35^{\mathrm{ab}}$ & $102.28^{\mathrm{ab}}$ & $1.75^{\mathrm{a}}$ & $326.6^{\mathrm{b}}$ \\
\hline $\begin{array}{l}\mathrm{T}_{8}: \mathrm{T}_{4}+\text { foliar application of } \mathrm{KH}_{2} \mathrm{PO}_{4} @ \\
1 \% \text { at } 60,80 \& 100 \mathrm{DAS}\end{array}$ & $158.7^{\mathrm{a}}$ & $2.2^{\mathrm{a}}$ & $27.3^{\mathrm{ab}}$ & $12.26^{\mathrm{a}}$ & $74.61^{\mathrm{a}}$ & $104.77^{\mathrm{a}}$ & $1.78^{\mathrm{a}}$ & $330.6^{\mathrm{ab}}$ \\
\hline $\begin{array}{l}\mathrm{T}_{9}: \mathrm{T}_{7}+\text { Soil mulching through polythene } \\
\text { sheet }(200 \text { gauge })\end{array}$ & $159.2^{\mathrm{a}}$ & $2.0^{\mathrm{a}}$ & $27.7^{\mathrm{a}}$ & $12.34^{\mathrm{a}}$ & $75.18^{\mathrm{a}}$ & $105.21^{\mathrm{a}}$ & $1.81^{\mathrm{a}}$ & $347.0^{\mathrm{a}}$ \\
\hline S.Em \pm & 3.4 & 0.34 & 0.9 & 0.38 & 1.92 & 2.03 & 0.08 & 6.28 \\
\hline $\operatorname{LSD}(0.05)$ & 13.2 & NS & 2.7 & 1.15 & 5.77 & 6.08 & 0.24 & 18.6 \\
\hline
\end{tabular}

Means with same letter do not differ significantly as per DMRT

DAS- Days after sowing TDM-Total dry matter production 
Table.2 Yield and yield attributes as influenced by different agronomic practices in $B t$ cotton

\begin{tabular}{|c|c|c|c|c|}
\hline Treatment & No. of bolls Plant ${ }^{-1}$ & Boll weight (g) & Seed cotton yield $\left(\mathrm{g}_{\text {plant }}{ }^{-1}\right)$ & Seed cotton yield $\left(\mathrm{kg} \mathrm{ha}^{-1)}\right.$ \\
\hline $\mathrm{T}_{1}$ : Normal spaced $(90 \mathrm{~cm} \mathrm{X} 60 \mathrm{~cm})$ non- $B t$ hybrid cv.RAHH-455. & $35.37^{\mathrm{e}}$ & $4.94^{b}$ & $130.1^{\mathrm{e}}$ & $2365^{\mathrm{d}}$ \\
\hline $\mathrm{T}_{2}$ : Normal planting $(90 \mathrm{~cm} \mathrm{X} 60 \mathrm{~cm}) \mathrm{Bt}$ hybrid Bindas & $40.83^{\mathrm{d}}$ & $5.04^{\mathrm{b}}$ & $140.7^{\mathrm{de}}$ & $2505^{\mathrm{d}}$ \\
\hline $\mathrm{T}_{3}$ : Closer spacing $(90 \mathrm{~cm} \mathrm{X} 45 \mathrm{~cm}) \mathrm{Bt}$ hybrid Bindas & $38.97^{\mathrm{de}}$ & $5.01^{\mathrm{b}}$ & $139.9^{\mathrm{de}}$ & $3189^{\mathrm{c}}$ \\
\hline $\begin{array}{l}\mathrm{T}_{4}: \mathrm{T}_{3}+125 \% \text { of Rec. Nutrients }(187.5: 92.75: \\
\left.92.75 \mathrm{~kg} \mathrm{~N}: \mathrm{P}_{2} \mathrm{O}_{5}: \mathrm{K}_{2} \mathrm{O} / \mathrm{ha}\right)\end{array}$ & $41.50^{\mathrm{cd}}$ & $5.11^{\mathrm{b}}$ & $142.7^{\mathrm{c}-\mathrm{e}}$ & $3268^{b c}$ \\
\hline $\begin{array}{l}\mathrm{T}_{5}: \mathrm{T}_{4}+\text { Recommended foliar spray }\left(1 \% \text { spray } \mathrm{MgSO}_{4} \text { at }\right. \\
90 \text { \& } 110 \mathrm{DAS} \text { and } 1 \% \text { spray of } \mathrm{KNO}_{3} \text { during } \\
\text { flowering \& Boll initiation stage })\end{array}$ & $42.97^{\mathrm{b}-\mathrm{d}}$ & $5.20^{\mathrm{ab}}$ & $145.5^{\mathrm{c}-\mathrm{e}}$ & $3314^{\mathrm{bc}}$ \\
\hline $\begin{array}{l}\mathrm{T}_{6}: \mathrm{T}_{4}+\text { Soil application of Micronutrients @ } 25 \mathrm{~kg} \\
\mathrm{MgSO}_{4} / \mathrm{ha}+10 \mathrm{~kg} \mathrm{FeSO}_{4}+10 \mathrm{~kg} \mathrm{ZnSO}{ }_{4} / \mathrm{ha} .\end{array}$ & $43.10^{\mathrm{b}-\mathrm{d}}$ & $5.25^{\mathrm{ab}}$ & $151.9^{\mathrm{b}-\mathrm{d}}$ & $3673^{\mathrm{a}-\mathrm{c}}$ \\
\hline $\begin{array}{l}\mathrm{T}_{7}: \mathrm{T}_{4}+\text { Three sprays of } 1 \% \mathrm{MgSO}_{4}+1 \% 19: 19: 19 \text { during } \\
60-65,80-85 \& 100-105 \mathrm{DAS}\end{array}$ & $45.67^{\mathrm{a}-\mathrm{c}}$ & $5.29^{\mathrm{ab}}$ & $157.3^{\mathrm{a}-\mathrm{c}}$ & $3777^{\mathrm{ab}}$ \\
\hline $\begin{array}{l}\mathrm{T}_{8}: \mathrm{T}_{4}+\text { foliar application of } \mathrm{KH}_{2} \mathrm{PO}_{4} @ 1 \% \text { at } 60,80 \\
\& 100 \text { DAS }\end{array}$ & $46.23^{\mathrm{ab}}$ & $5.44^{\mathrm{ab}}$ & $163.8^{\mathrm{ab}}$ & $3912^{\mathrm{a}}$ \\
\hline $\mathrm{T}_{9}: \mathrm{T}_{7}+$ Soil mulching through polythene sheet (200 gauge) & $48.27^{\mathrm{a}}$ & $5.64^{\mathrm{a}}$ & $170.8^{\mathrm{a}}$ & $4122^{\mathrm{a}}$ \\
\hline S.Em \pm & 1.33 & 0.15 & 5.00 & 162 \\
\hline $\operatorname{LSD}(0.05)$ & 3.99 & 0.45 & 14.9 & 487 \\
\hline
\end{tabular}

Means with same letter do not differ significantly as per DMRT DAS- Days after sowing

Table.3 Leaf reddening index (LRI) of $B t$ cotton at different growth stages as influenced by different agronomic practices

\begin{tabular}{|c|c|c|c|c|}
\hline \multirow{2}{*}{\multicolumn{2}{|c|}{ Treatment }} & \multicolumn{3}{|c|}{ Leaf reddening index (LRI) } \\
\hline & & \multirow{2}{*}{$\frac{\text { 90 DAS }}{0.71^{\mathrm{a}}}$} & \multirow{2}{*}{$\frac{135 \text { DAS }}{2.33^{\mathrm{a}}}$} & \multirow{2}{*}{$\frac{\text { At final Picking }}{2.93^{\mathrm{a}}}$} \\
\hline $\mathrm{T}_{1:}$ & Normal spaced $(90 \mathrm{~cm}$ X $60 \mathrm{~cm})$ non- $B t$ hybrid cv.RAHH-455 & & & \\
\hline $\mathrm{T}_{2:}$ & Normal planting $(90 \mathrm{~cm}$ X $60 \mathrm{~cm}) B t$ hybrid Bindas & $0.62^{\mathrm{a}}$ & $2.02^{\mathrm{ab}}$ & $2.87^{\mathrm{a}}$ \\
\hline $\mathrm{T}_{3:}$ & Closer spacing $(90 \mathrm{~cm} \mathrm{X} 45 \mathrm{~cm}) \mathrm{Bt}$ hybrid Bindas & $0.55^{\mathrm{a}}$ & $1.87^{\mathrm{ab}}$ & $2.73^{\mathrm{ab}}$ \\
\hline $\mathrm{T}_{4:}$ & $\mathrm{T}_{3}+125 \%$ of Rec. nutrients $(187.5: 92.7: 92.7 \mathrm{~kg} \mathrm{NPK} / \mathrm{ha})$ & $0.50^{\mathrm{a}}$ & $1.80^{\mathrm{ab}}$ & $2.60^{\mathrm{a}-\mathrm{c}}$ \\
\hline $\mathrm{T}_{5}$ & $\begin{array}{l}\mathrm{T}_{4}+\text { Recommended foliar spray ( } 1 \% \text { spray } \mathrm{MgSO}_{4} \text { at } 90 \& 110 \mathrm{DAS} \text { and } 1 \% \text { spray of } \mathrm{KNO}_{3} \\
\text { during flowering \& Boll initiation stage) }\end{array}$ & $0.43^{\mathrm{a}}$ & $1.66^{\mathrm{ab}}$ & $2.57^{\mathrm{a}-\mathrm{c}}$ \\
\hline $\mathrm{T}_{6:}$ & $\mathrm{T}_{4}+$ Soil application of Micronutrients @ $25 \mathrm{~kg} \mathrm{MgSO}_{4} / \mathrm{ha}+10 \mathrm{~kg} \mathrm{FeSO}_{4}+10 \mathrm{~kg} \mathrm{ZnSO} / \mathrm{ha}$ & $0.40^{\mathrm{a}}$ & $1.43^{\mathrm{ab}}$ & $2.40^{\mathrm{a}-\mathrm{d}}$ \\
\hline $\mathrm{T}_{7:}$ & $\mathrm{T}_{4}+3$ sprays of $1 \% \mathrm{MgSO}_{4}+1 \% 19: 19: 19$ during $60-65,80-85 \& 100-105 \mathrm{DAS}$ & $0.35^{\mathrm{a}}$ & $1.40^{\mathrm{ab}}$ & $2.20^{\mathrm{b}-\mathrm{d}}$ \\
\hline $\mathrm{T}_{8}$ & $\mathrm{~T}_{4}+$ foliar application of $\mathrm{KH}_{2} \mathrm{PO}_{4} @ 60,80$ \&100DAS & $0.27^{\mathrm{a}}$ & $1.37^{\mathrm{ab}}$ & $2.04^{\mathrm{cd}}$ \\
\hline \multirow[t]{3}{*}{$\mathrm{T}_{9}$} & $\mathrm{~T}_{7}+$ Soil mulching through polythene sheet (200 gauge) & $0.20^{\mathrm{a}}$ & $1.07^{\mathrm{b}}$ & $1.93^{\mathrm{d}}$ \\
\hline & S.Em \pm & 0.05 & 0.14 & 0.21 \\
\hline & $\operatorname{LSD}(0.05)$ & 0.15 & 0.42 & 0.62 \\
\hline
\end{tabular}

Means with same letter do not differ significantly as per DMRT. DAS-Days after sowing 
Table.4 Monetary returns of $B t$ cotton as influenced by different agronomic practices

\begin{tabular}{|c|c|c|c|c|}
\hline Treatment & Cost of cultivation $\left(\square \mathbf{h a}^{-1}\right.$ ) & Gross returns $\left(\square\right.$ ha $\left.^{-1}\right)$ & Net returns $\left(\square\right.$ ha $\left.^{-1}\right)$ & B:C ratio \\
\hline $\mathrm{T}_{1}$ : Normal spaced $(90 \mathrm{~cm} \mathrm{X} 60 \mathrm{~cm})$ non- $B t$ hybrid cv.RAHH-455. & 54955 & 127692 & 72737 & $2.32^{\mathrm{d}}$ \\
\hline $\mathrm{T}_{2}:$ Normal planting $(90 \mathrm{~cm} \mathrm{X} 60 \mathrm{~cm}) \mathrm{Bt}$ hybrid Bindas & 56715 & 135288 & 78573 & $2.39^{\mathrm{d}}$ \\
\hline $\mathrm{T}_{3}$ : Closer spacing $(90 \mathrm{~cm} \mathrm{X} 45 \mathrm{~cm}) \mathrm{Bt}$ hybrid Bindas & 57265 & 172217 & 114952 & $3.01^{\mathrm{bc}}$ \\
\hline $\mathrm{T}_{4}: \mathrm{T}_{3}+125 \%$ of Rec. Nutrients (187.5: $\left.92.75: 92.75 \mathrm{~kg} \mathrm{~N}: \mathrm{P}_{2} \mathrm{O}_{5}: \mathrm{K}_{2} \mathrm{O} / \mathrm{ha}\right)$ & 59443 & 176466 & 117023 & $2.97^{\mathrm{bc}}$ \\
\hline $\begin{array}{l}\mathrm{T}_{5}: \mathrm{T}_{4}+\text { Recommended foliar spray }\left(1 \% \text { spray } \mathrm{MgSO}_{4} \text { at } 90 \& 110 \mathrm{DAS} \text { and }\right. \\
\left.1 \% \text { spray of } \mathrm{KNO}_{3} \text { during flowering \& Boll initiation stage }\right)\end{array}$ & 61060 & 178947 & 117887 & $2.93^{c}$ \\
\hline $\begin{array}{l}\mathrm{T}_{6}: \mathrm{T}_{4}+\text { Soil application of Micronutrients @ } 25 \mathrm{~kg} \mathrm{MgSO}_{4} / \mathrm{ha}+10 \mathrm{~kg} \mathrm{FeSO}_{4}+ \\
10 \mathrm{~kg} \mathrm{ZnSO}_{4} / \mathrm{ha} \text {. }\end{array}$ & 61907 & 198348 & 136441 & $3.20^{\mathrm{abc}}$ \\
\hline $\begin{array}{l}\mathrm{T}_{7}: \mathrm{T}_{4}+\text { Three sprays of } 1 \% \mathrm{MgSO}_{4}+1 \% 19: 19: 19 \text { during 60-65, 80-85 \& } \\
100-105 \text { DAS }\end{array}$ & 59657 & 203959 & 142832 & $3.34^{\mathrm{ab}}$ \\
\hline $\mathrm{T}_{8}: \mathrm{T}_{4}+$ foliar application of $\mathrm{KH}_{2} \mathrm{PO}_{4} @ 1 \%$ at $60,80 \& 100 \mathrm{DAS}$ & 60542 & 211248 & 150706 & $3.49^{\mathrm{a}}$ \\
\hline $\mathrm{T}_{9}: \mathrm{T}_{7}+$ Soil mulching through polythene sheet (200 gauge) & 63292 & 222588 & 159296 & $3.52^{\mathrm{a}}$ \\
\hline S.Em \pm & & 14081 & 10378 & 0.12 \\
\hline $\operatorname{LSD}(0.05)$ & & 42252 & 31115 & 0.37 \\
\hline
\end{tabular}

Means with same letter do not differ significantly as per DMRT. DAS-Days after sowing

Fig.1 Effect of soil temperature variation between mulched and unmulched treatments

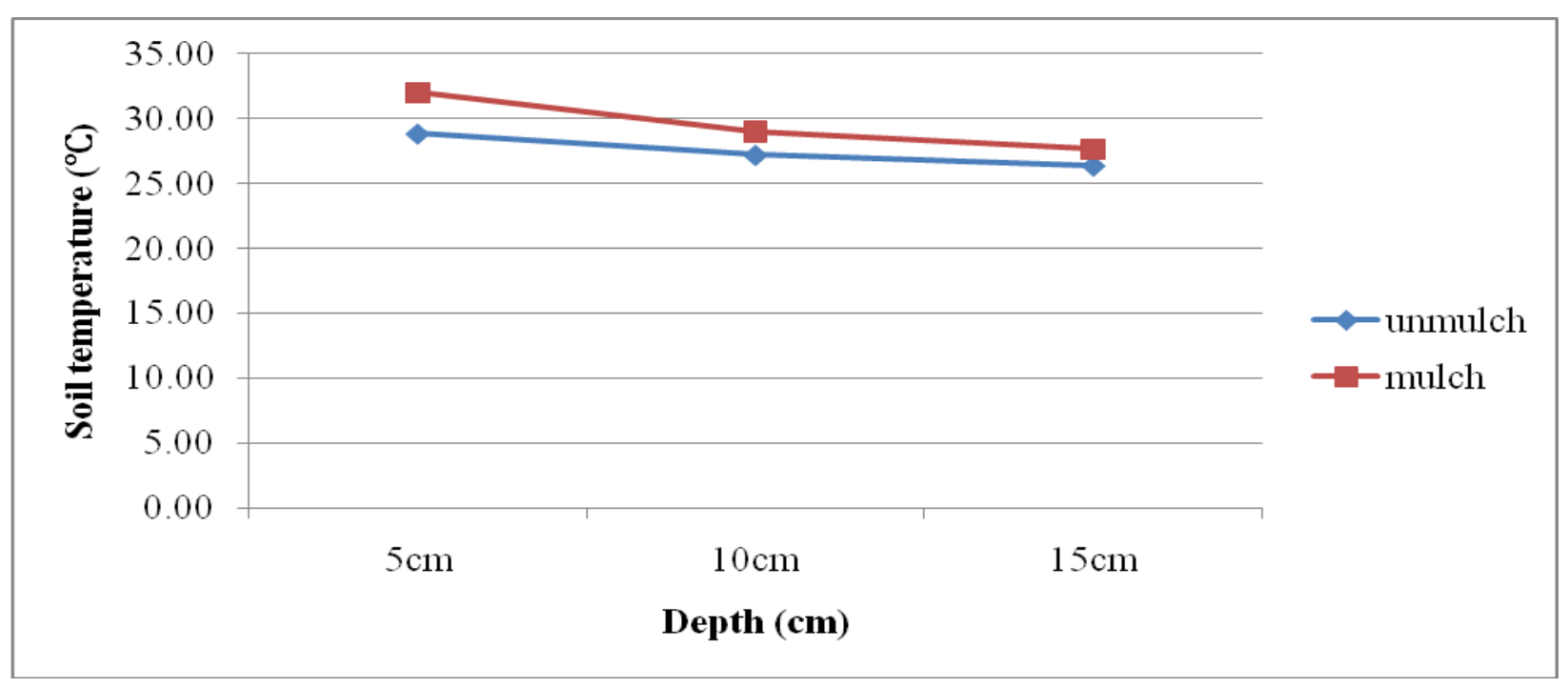




\section{Economics}

Consequent upon higher yield, gross ( $\square$ 2,22,588 $\left.\mathrm{ha}^{-1}\right)$ and net ( $\square$ 1,59,296 hä) returns and B:C (3.52) were significantly higher with closer spacing with $125 \% \mathrm{RDF}$ and three sprays of $1 \%$ each of $\mathrm{MgSO}_{4}$ and 19:9:19 at 60,80 and 100 DAS with PE mulching $\left(\mathrm{T}_{9}\right)$.

Further the gross and net returns and B: C reached the minimum $(\square$ 1,27,692 and 72,737 $\mathrm{ha}^{-1}$ and 2.32, respectively) with normal spaced non- $B t \mathrm{cv}$. RAHH-445 $\left(\mathrm{T}_{1}\right)$.

This is on the expected line as higher the nutrition to the crops higher will be the cotton yield and it ultimately lead to higher gross and net returns. The results are in conformity with Kaur et al., (2010), Biradar et al., (2011), Basavanneppa (2012) and Hosamani et al., (2013) who also obtained higher net returns with increased fertilizer levels.

From the study, it was inferred that, $B t$ cultivar with $25 \%$ higher population $(90 \mathrm{~cm}$ $\mathrm{X} 45 \mathrm{~cm})$ and $125 \% \mathrm{RDF}(187.5: 92.75: 92.75$ $\left.\mathrm{kg} \quad \mathrm{N}: \quad \mathrm{P}_{2} \mathrm{O}_{5}: \quad \mathrm{K}_{2} \mathrm{O} \quad \mathrm{ha}^{-1}\right)$ is monetarily advantageous in Tunga Bhadra Project (TBP) irrigation command. Further, leaf reddening management practice through foliar nutrition of 1\% each of $\mathrm{MgSO}_{4}$ and 19:19:19 thrice (60-65, 80-85 and 100-105 DAS) and $\mathrm{KNO}_{3}$ twice (during flowering, boll initiation stage and boll bursting stages) and soil mulching with polyethylene mulch would further enhance yield and monetary returns (Table 4 ). Alternately, $1 \% \mathrm{KH}_{2} \mathrm{PO}_{4}$ thrice $(60,80$ and 100 DAS) could be adopted.

\section{Acknowledgement}

To the University agricultural sciences, College of Agriculture Raichur for provision of research facilities is gratefully acknowledged.

\section{References}

Anonymous, 2014, Annual report. All India Coordinated Cotton Improvement Project.

Basavanneppa, M. A. 2012. Effect of nutrients on endotoxin and management of refuge crops in $B t$ cotton under irrigated condition. Ph. D. Thesis, Uni. Agric. Sci., Dharwad, Karnataka (India).

Basavanneppa, M. A., Biradar, D. P, Ajay Kumar, M. Y and Shivakumar. 2011. Influence of foliar nutrition on $B t$ cotton productivity and profitability in irrigated ecosystem. World Cotton Res. Conf. on Technol. for Prosperity-5, Mumbai, 7-11 November 2011, Book of Abstracts, p. 156.

Biradar, D. P., Aladakatti, R, Satyanarayana, T and Shivamurthy, D. 2012. Enhancing the productivity and profitability of $B t$ cotton hybrids through site specific nutrient management and optimum planting geometry, In: $3^{\text {rd }}$ Intl. Agron. Congress., 26-30 November, New Delhi, Vol., 3: pp. 851-852.

Biradar, D. P., Aladakatti, Y. R., Basavanneppa, M. A, Shivamurthy, D and Satyanarayana, T. 2011. Assessing the contribution of nutrients to maximize transgenic cotton yields in vertisols of Northern Karnataka. Better Crops, 5(1): 22-25.

Brar, M. S., Gill, M. S, Sekhan, K. S, Sidhu, B. S, Sharma, P and Singh, A. 2008. Effect of soil and foliar application of nutrients on yield and nutrient concentration in $B t$ cotton. J. Res., Punjab Agric. Univ., 45 (3\&4) : 126131.

Dilip Kumar, G., Sachin, S. S and Rajesh Kumar. 1990. Importance of mulch in crop production. Indian J. Soil Cons., 18: 20-26.

Honnalli, S. N and Chittapur, B. M. 2013. 
Enhancing Bt Cotton (Gossypium spp.) productivity through transplanting in Upper Krishna Project (UKP) command area of Karnataka. Indian J. Agron., 58(1): 105-108.

Hosamani, V., Halepyati, A. S, Koppalkar, B. G, Desai, B. K and Ravi, M. V. 2013. Yield, quality parameters and uptake of nutrients in irrigated $B t$ cotton (Gossypium hirsutum L.) as influenced by macro nutrients and liquid fertilizers. Karnataka J. Agric. Sci., 26(3): 421423.

Hosmath, J. A.,2011. Evaluation of Bt cotton genotypes and nutrient management to control leaf reddening. Ph. D. Thesis, Univ. Agric. Sci., Dharwad, Karnataka.

Kaur, P., Kaur, M, Gill, M. S and Buttar, G. S. 2010. Response of $B t$ cotton hybrid $\mathrm{RCH}-134$ to varied spacing and fertility levels under Punjab conditions. $J$. Cotton Res. Dev., 24(2): 189-192.

Mamatha, N., Bidari, B. I, Shashidhar, G. B and Channal, H. T. 2009. Yield and quality of cotton (Gossypium hirsutum L.) as influenced by sulphur and micronutrients. An Asian J. Soil Sci. 4: $71-73$

Rajendran, K., Mohamed, M. A and Vaiyapuri, K. 2011. Influence on growth, yield attributes and yield of $B t$ cotton by soil and foliar application of nutrients. Madras Agric. J., 98(1-3): 67-
68.

Rajput, R. K and Singh, M. 1970. Efficiency of different mulches on conserving soil moisture in cotton. Inian. J. Agron., 15: 41-45.

Sakarvadia, H. L., Polara, K. B, Parmar, K. B, Babariya, N. B and Kunadia, B. B. 2009. Effect of potassium and zinc on growth, yield, quality parameters and nutrient uptake by $B t$ cotton. Asian $J$. Soil Sci., 4(1): 24-26.

Saleem, M. F., Bilal, M. F, Awais, M, Shahid, N. Q and Anjum, S. A. 2010. Effect of nitrogen on seed cotton yield and fiber qualities of $B t$ cotton cultivars. $J$. Animal Plant Sci., 20(1): 23-27.

Sawan, Z. M., Mahmoud, $\mathrm{H}$ and El Guibali Amal, H. 2006. Response of yield, yield components and fibre properties of $B t$ cotton to nitrogen fertilization and foliar applied potassium and mepiquat chloride. J. Cotton Sci., 10: 224-234.

Shivamurthy, D and Biradar, D. P. 2014. Effect of foliar nutrition on growth, yield attributes and seed cotton yield of Bt cotton. Karnataka J. Agric. Sci. 27(1): 5-8.

Yadav, R. L and Meena, M. C. 2009. Available micronutrients status and their relationship with soil properties of Degana soil series of Rajasthan. $J$. Indian Soc. Soil Sci. 57: 90-92.

\section{How to cite this article:}

Basavaraj, B.M. Chittapur, A.S. Halepyati, A. Ameregouda, G.S. Yadahalli and Ajayakumar, M.Y. 2017. Growth and Yield of Irrigated Bt Cotton (Gossypium hirsutum L.) as Influenced by Different Agronomic Practices. Int.J.Curr.Microbiol.App.Sci. 6(12): 45-52. doi: https://doi.org/10.20546/ijcmas.2017.612.006 